\title{
Poorly Differentiated Adenocarcinoma Manifesting as Multifocal Obstructive Disease -A Rare Occurrence
}

\author{
Pragati Aditya Sathe, Jayashri Popat Chaudhari*, Manjusha Milind Karegar and Amit Suresh Mirjolkar \\ Department of Pathology, Seth G. S. Medical College \& KEM Hospital, Mumbai, India
}

\begin{abstract}
We describe an autopsy of a very unusual case of an adult female presenting with multifocal obstructive symptoms like achalasia, obstructive jaundice and bilateral hydronephrosis . Primary clinical diagnosis was tuberculosis and malignancy. Although the patient was extensively worked up in the hospital with multiple biopsies, the diagnosis could not be made. Autopsy revealed contiguous involvement of entire gastrointestinal tract from esophagus till colon by a poorly differentiated adenocarcinoma with diffuse thickening, firmness and rigidity of the wall. The tumor cells were focally expressing CK 20 favouring a colonic primary. Colonic adenocarcinoma usually spread by either lymphatic or hematogenous way to lymph nodes, liver and lung. This unusual way of spread of this tumor contiguously from colon till esophagus, with Urinary bladder and distal CBD infiltration make it very unique.
\end{abstract}

Keywords: Visceral Carcinomatosis, Multifocal Obstruction, Adenocarcinoma

\section{Introduction}

Adenocarcinoma of colon usually metastasizes through lymphatics or hematogenously and the common sites are the regional lymph nodes, liver and lung. ${ }^{[1]}$ Unusual sites of metastasis include skin, skeletal muscle, bone and other visceral organs. ${ }^{[1]}$ Colonic cancer presenting as widespread submucosal metastasis to multiple organs like the esophagus, stomach, small intestine, distal common bile duct and the urinary bladder is quite uncommon. We report a rare case of disseminated malignancy of colon presenting deceptively as pseudoachalasia, obstructive jaundice and bilateral hydronephrosis.

\section{Case Report}

A 40- year-old female, complained of decreased appetite, weight loss and hematuria for two months. Subsequently she developed diarrhea, dysphagia for solids followed by liquids and progressive jaundice with itching for a period of one month. On general examination, the vital parameters were normal. The patient was cachectic, had marked pallor and icterus with no lymphadenopathy. Respiratory, cardiovascular system and per abdominal examination did not reveal any abnormality.

In the prolonged ward stay of 37 days, she had progressive pancytopenia. Her hemoglobin declined from $12.0 \mathrm{gm} / \mathrm{dl}$ to $7.9 \mathrm{gm} / \mathrm{dl}$, Total leukocyte count decreased from 16700/ $\mathrm{cmm}$ to $3400 / \mathrm{cmm}$ and platelet count decreased from 3.6 lac to $1.2 \mathrm{lac} / \mathrm{cmm}$. Serum creatinine level increased from $1.5 \mathrm{mg} / \mathrm{dl}$ to $5.8 \mathrm{mg} / \mathrm{dl}$. Liver function tests revealed maximum total bilirubin was $4.3 \mathrm{mg} / \mathrm{dl}$, and direct bilirubin - $3.4 \mathrm{mg} / \mathrm{dl}$, alkaline phosphatise - $880 \mathrm{U} / \mathrm{L}$, alanine aminotransferase $-45 \mathrm{IU} / \mathrm{L}$ and aspartate aminotransferase - $50 \mathrm{IU} / \mathrm{L}$. Stool examination showed presence of occult blood. Computed Tomography of abdomen showed thickening of urinary bladder, uretero-vesicle junction obstruction, bilateral hydronephrosis, stricturous thickening of terminal common bile duct and thickening of gastric wall and diffuse thickening of the small and large intestinal wall. Barium swallow revealed distal esophageal stricture. Esophageal Manometry revealed grade III achalasia cardia. Endoscopic ultrasonography showed long segment esophageal narrowing with gastric and duodenal thickening possibly due to an inflammatory or a neoplastic process. Biopsy from the gastric body and antrum did not show malignancy. Sigmoidoscopy showed bleeding haemorrhoids. Urinary bladder biopsy too was inadequate and did not contribute to diagnosis. Urine cytology did not show any malignant cells. Serum level of CA125 was 336.8 $\mathrm{U} / \mathrm{ml}$ (Normal range 0-37 unit $/ \mathrm{ml}$ ) and serum CA19.9 was $633.8 \mathrm{U} / \mathrm{ml}$ (Normal range 0-37 unit/ml) while serum alpha fetoprotein and carcinoembryonic antigen levels were normal. Serum levels of anti smooth muscle antibody were also normal. The clinical impression was achalasia cardia, gastroenteritis and obstructive jaundice possibly due to tuberculosis or malignancy. As no definite diagnosis could be established, the patient continued to receive symptomatic treatment. Further, during the ward stay she exhibited altered behaviour with irrelevant speech and the symptoms were attributed to metabolic encephalopathy. As a palliative procedure, percutaneous transhepatic biliary drainage was performed. Later, the patient developed oliguria with further deterioration of sensorium 
and succumbed to the illness. A complete autopsy was performed to ascertain the cause of death.

Autopsy Findings: Externally, there was pallor and icterus without lymphadenopathy. Breast examination was normal. On systemic examination, both lungs showed patchily thickened pleura and few small calcified areas in the upper lobe on cut surface. In the abdominal cavity, $200 \mathrm{cc}$ of yellow hazy fluid was noted. . The proximal esophagus was dilated. There was diffuse thickening and rigidity of the wall of distal esophagus, stomach, small and large intestine with multiple strictures. (Figure 1) The cut surface appeared firm, variably thickened to a maximum thickness of one centimeter and had glistening appearance. The mucosal folds were enhanced in some areas. The periampullary region was thickened with narrowing of the distal common bile duct. (Figure 1) Both kidneys showed hydronephrosis with thinned out renal parenchyma. (Figure 1) The urinary bladder was shrunken with rigidity of the wall and narrowed cavity. The cut surface was similar to that of the GI tract. (Figure 1) Both ureters were dilated. Few enlarged mesenteric lymph nodes with glistening cut surface were seen, the largest measuring 1.5 centimeters in size.
Histopathology Features: Esophagus, gastro esophageal junction, stomach, the small intestine and the large intestine showed diffuse infiltration by singly scattered tumor cells with round vesicular nuclei and prominent nucleoli amidst desmoplastic stroma. (Figure 2) Few cells had classic signet ring cell morphology. Gland formation was very focally visualised on studying multiple sections. The tumor was primarily seen in the submucosa but also infiltrated the lamina propria, muscularis propria and the subserosal adipose tissue. The mucosa of the gastroesophageal junction, stomach, small and the large intestine did not reveal any dysplasia despite multiple sections. The mesenteric lymph nodes showed metastatic carcinoma. The tumor cells expressed CK 20 but were negative for CK 7 suggesting a primary colonic poorly differentiated adenocarcinoma. (Figure 2) The common bile duct and urinary bladder also showed diffusely scattered tumor cells with marked desmoplasia within the wall. However, the mucosa did not reveal any dysplastic changes. Both kidneys showed focal interstitial fibrosis. The lungs showed foci of healed tuberculosis. The omentum showed tumor deposits. (Figure 2) Multiple tumor emboli were seen in the uterus and both ovaries. (Figure 2) Metastatic foci were not seen in the liver and the lungs.

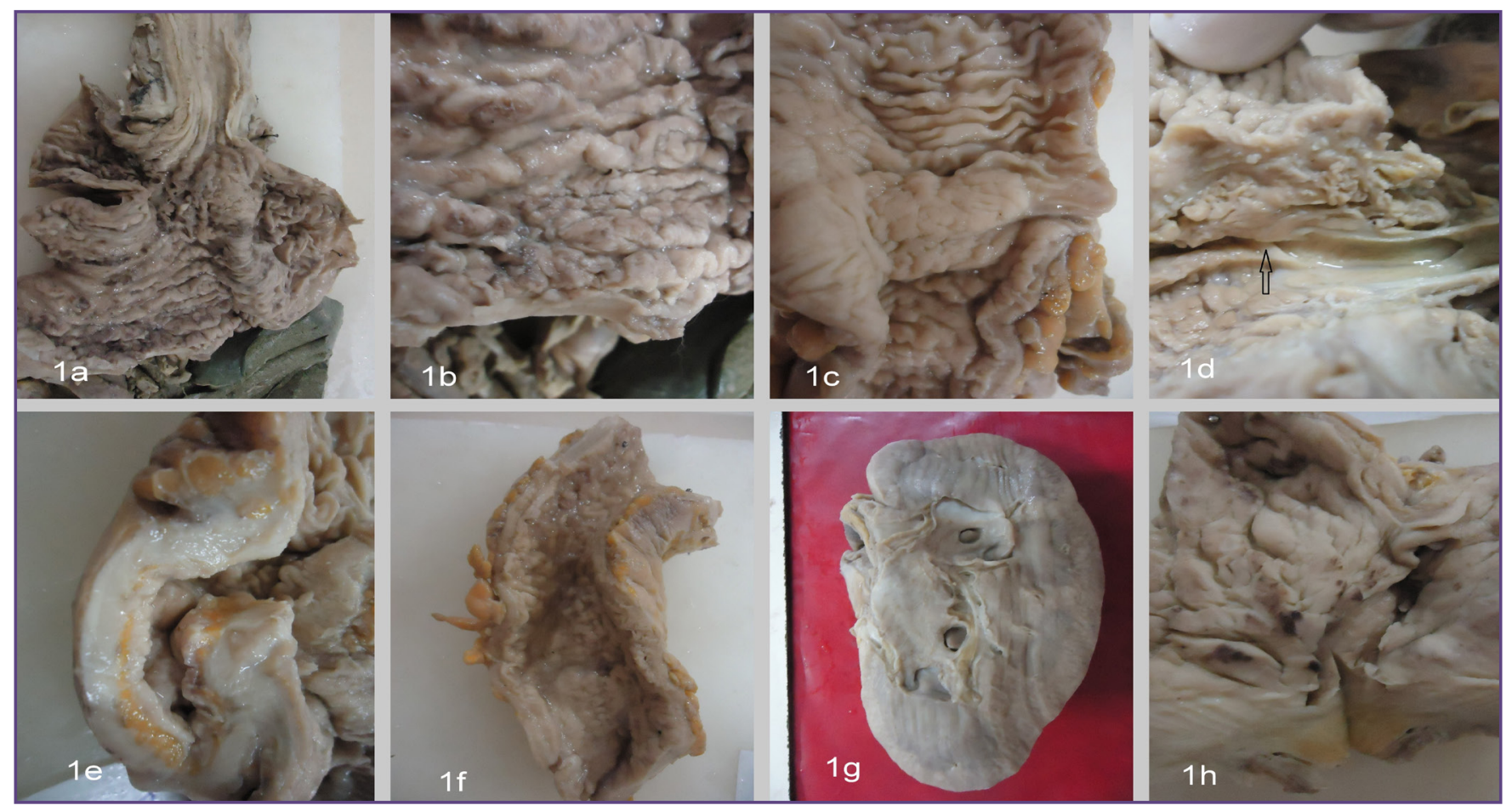

Fig. 1: Gross findings: a) GE junction showing thickened wall of lower esophagus and narrowing. b) Closer view of gastric mucosa showing prominent rugosity c) Small intestine showing transverse stricture and flattening of mucosa d) Common bile duct showing distal thickening (arrow) and proximal dilatation e) Cut surface of cecal wall showing thickened glistening wall f) Diffusely thickened narrow rigid colon with glistening wall g) Kidney showing mild hydronephrosis h) Urinary bladder showing diffusely thickened wall with prominence of mucosal folds 


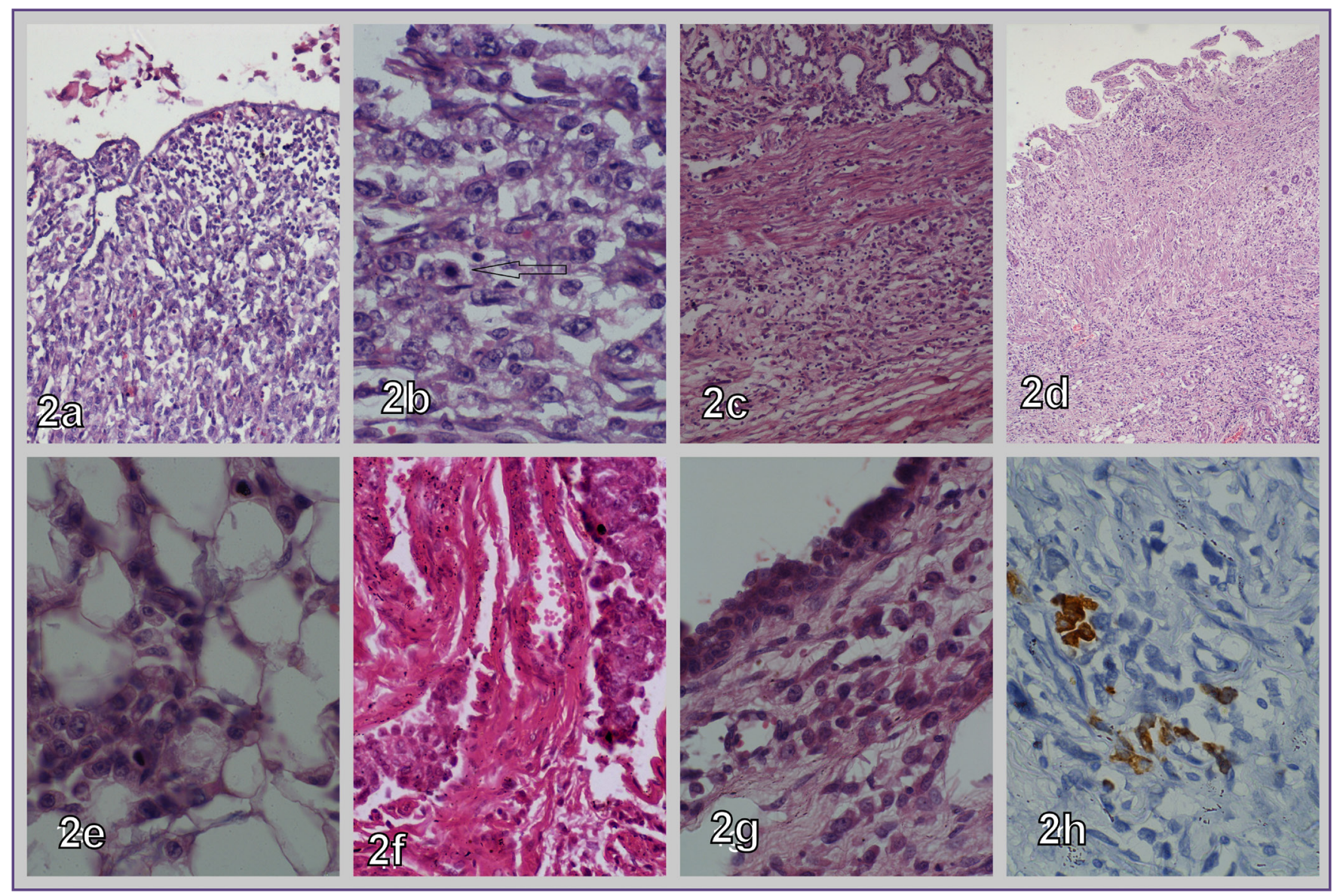

Figure 2: Microscopy: a) Colonic mucosa showing ulceration and diffuse infiltration by tumor cells. (HE,x100) b) Colon: mitotic figure is show by arrow. (HE, $x$ 600) c) Gastroesophageal junction showing similar cells (HE,x100) d) Small intestine showing full thickness infiltration(HE, x40) e) Omentum showing infiltration by tumor cells. (HE, $x$ 400) $f$ ) Uterine myometrium showing lymphatic tumor emboli . (HE, x 400) g) Urinary bladder wall showing similar cells. (HE, $x$ 400) h) Tumor cells showing focal but strong positivity for (CK $20 \times 400$ )

\section{Discussion}

The death was attributed to disseminated carcinomatosis conspicuously involving the hollow visceral organs. The middle aged woman discussed above presented with obstructive pathology involving the esophagus, biliary tree and the urinary tract The differential diagnoses entertained were malignancy (hematological or non-hematological) and tuberculosis. Disseminated high grade Non Hodgkin lymphoma or MALT lymphoma may present with such extensive multi organ involvement. ${ }^{[2]}$ However, these tumors usually affect spleen, liver, lymph nodes and bone marrow which was not seen in our case. Also, grossly, the thickened wall would have been fleshy and softer in consistency. Similarly, disseminated abdominal tuberculosis shows multiple lymphadenopathy, hepatic, splenic and peritoneal involvement. Absence of caseous necrosis or milliary nodules typical of the disease ruled out this possibility.
The unusual feature in our case was the hose-pipe like rigidity with thickened wall of multiple gastrointestinal and non gastrointestinal organs. On cut surface, the glistening and firm appearance of the wall favored a diagnosis of mucin producing carcinoma with reactive desmoplasia. On microscopy, the diagnosis of poorly differentiated adenocarcinoma was confirmed. However, another challenge was to find the primary tumor. Expression of CK 20 by tumor cells and negativity for CK 7 favored a colonic primary lesion over stomach or common bile duct though we could not specify the exact location in the colon ${ }^{[3]}$ It is usual for colonic carcinoma to involve the urinary bladder by direct extension but distant metastasis to the wall of the urinary bladder is unusual. ${ }^{[4]}$ Of the two morphologic subtypes of metastatic adenocarcinomas to the urinary bladder, namely protuberant and diffuse, the diffuse type is difficult to diagnose as the presentation can be subtle..$^{[5]}$ 
This type presents with hydronephrosis and diffuse bladder wall thickening as seen in our case.

Extrahepatic bile duct obstruction in primary colonic or gastric tumors is known and is usually secondary to external compression by metastatic peripancreatic or periportal lymphnodes. ${ }^{[6]}$ However, mural involvement of extrahepatic bile duct by a metastatic carcinoma leading to obstructive jaundice is quite uncommon.

Themostcommon cause of pseudoachalasia is a compressing extraluminal mass or an intramural involvement by gastric adenocarcinoma at the gastroesophageal junction. [7] Thorough investigation with endoscopic ultrasound and guided biopsies are recommended if malignancy is suspected. However, if the tumor is mainly submucosal and has desmoplastic stroma, a superficial biopsy may be inconclusive, as it happened in our case. The sparing of liver and lungs could be attributable to micrometastasis as thought in previous reports. ${ }^{[1]}$

The exact mechanism of distant metastasis in our case is not known. Subperitoneal space act as a highway for lymphatics and blood vessels. Tumors can also spread fast by this pathway. There are four different ways in which tumors can spread, either directly or through ascitic fluid, lymphatics or blood vessels. In direct spread, it can be contiguous or non-contiguous via the subperitoneal space along the ligaments, mesenteries and omenta. Tumors of the stomach, colon and pancreas commonly spread directly. Probably the direct contiguous spread could have resulted in contiguous involvement of entire gastrointestinal tract and incontiguous direct spread would explain urinary bladder involvement. ${ }^{[8]}$ Work on Glycosylation alterations have demonstrated role of glycobiology in cancer cell adhesion, signaling, invasion and metastasis. ${ }^{[9]}$ In our case, some such alteration may have occurred which could explain the unusual method of metastasis.

By performing autopsy and studying pathology in this case, it was concluded that such a varied presentation was contributed by a single disease. This has also highlighted the most unusual presentation of the usual adenocarcinoma and would help in achieving a successful management of patients in future.

\section{Conclusion}

Colonic carcinoma presenting as obstructive lesions in multiple hollow visceral organs without demonstrable involvement of liver and the lung is rare. Thus, poorly differentiated adenocarcinomas of the gastrointestinal tract may surprise clinicians and pathologists by their unusual behaviour. Keeping this in mind, a high index of suspicion is needed. As malignancy was always suspected in our case, repeat deeper biopsies may be from alternate site like the colon, would have probably clinched the diagnosis.

\section{References}

1. Attili VSS, Rama Chandra C, Dadhich HK, Sahoo TP, Anupama G, Bapsy PP. Unusual metastasis in colorectal cancer. Ind J Cancer 2006; 43:93-95.

2. Weng SC, Wu CY. Lymphoma presenting as peritoneal lymphomatosis with ascites. J Chin Med Assoc 2008 ; 71:646-50.

3. Park SY, Kim HS, Hong EK, Kim WH. Expression of cytokeratins 7 and 20 in primary carcinomas of the stomach and colorectum and their value in the differential diagnosis of metastatic carcinomas to the ovary. Hum Pathol 2002;33: 1078-85

4. Chang CL, Chen YT. Metastatic Colon Cancer to the Urinary Bladder: A Case Report J Soc Colon Rectal Surgeon (Taiwan) 2009;20:39-43

5. Sharma PK, Vijay MK, Das RK, Chatterjee U. Secondary signet-ring cell adenocarcinoma of urinary bladder from a gastric primary. Urol Ann. 2011;3: 97-99.

6. Lee DH, Ahn YJ, Shin R, Lee HW. Metastatic mucinous adenocarcinoma of the distal common bile duct, from transverse colon cancer presenting as obstructive jaundice. Korean J Hepatobiliary Pancreat Surg. 2015;19: 125-128

7. Campo SMA, Zullo A, Scandavini CM, Frezza B, Cerro P, Balducci G. Pseudoachalasia: A peculiar case report and review of the literature. World J Gastrointest Endosc 2013; 5: $450-454$

8. Le Ott. Patterns of peritoneal spread of tumor in the abdomen and pelvis. World J Radiol 2013 28; 5: 106-112

9. Mereiter S, Balmaña M, Gomes J, Magalhães A, Reis CA. Glycomic Approaches for the Discovery of Targets in Gastrointestinal Cancer. Front Oncol 2016;6:55.

*Corresponding author:

Dr Jayashri Chaudhari, Department of Pathology, 1st floor, College building, KEM Hospital, Mumbai -12, India

Phone: +91 9869793185

Email: jayashreepath@gmail.com

Financial or other Competing Interests: None. 